\title{
AEFI Reporting Rates in Albania During 2008-2015: A Retrospective Analysis of AEFI Reporting Forms
}

\author{
Irsida Mehmeti \\ Department of Pharmaceutical Sciences, Catholic University Our Lady of Good Counsel, \\ Silva Bino \\ Control of Infectious Diseases Department, Institute of Public Health, Tirana, Albania \\ Erida Nelaj \\ Control of Infectious Diseases Department, Institute of Public Health, Tirana, Albania \\ Eugena Tomini \\ Control of Infectious Diseases Department, Institute of Public Health, Tirana, Albania
}

Abstract

In Albania, passive AEFI surveillance is conducted by Institute of Public Health, which is under the supervision of Ministry of Health. Reports of suspected adverse events following immunisation (AEFI) are reviewed by the Control of infectious disease department in the Institute of Public Health and recorded in a central database. In this study AEFI database is analysed for individual AEFI reporting forms during 2008-2015. AEFI reporting rates and their patterns were assessed. At 2003 a national standardized AEFI reporting form was introduced to the health-care workers who were obligated to report any observed AEFI. This form was changed and added more requisites in 2008 and nowadays this is the formal AEFI reporting form in Albania. Safe vaccines are essential to preserve high adherence to immunization programs [1]. During the last years the reporting rate increased significantly due to implementation of enhanced vaccine safety reporting strategies but it is still low and underreporting remains one of the limits of passive surveillance. Reporting rates were calculated using as a denominator the administered doses of vaccines.

\section{Objective}

The first aim of this study is to individuate the principal patterns of adverse events following immunization in Albania since establishment of AEFI system till the last year and highlight any significant difference between these patterns. The second objective is to observe the AEFI reporting rate during the years and evaluate the possibility of undertaking new provisions to expand the reporting of $\mathrm{AEFI}$ and strengthen the rules of reporting the AEFI.

\section{Introduction}

The implementation of vaccine safety surveillance system in Albania has started since 2003 as part of National Immunization Program (NIP). A modified version of the WHO recommended reporting form with standard case definitions was adopted. Data received from individual reporting forms are collated in the National AEFi database situated in the Institute of Public Health. In 2008 the AEFI database was enriched with more information and details about each case. Brighton Collaboration case definitions of adverse events following immunization is adopted to code and define each medical terminology.

In Albania the surveillance of adverse events following immunization relies on passive surveillance and active surveillance conducted during mass immunization campaign. Reporting of AEFI is mandatory and is done by primary health care providers in public health services all over the country, tertiary health care providers in the national Universitary Hospital center "Mother Theresa" and a few private health care units. Detection, validation, investigation and causality assessment of vaccine safety signals are core objectives of Albanian National Institute of Public Health. Expert opinion is provided by AEFI Expert Committee situated at the Ministry of Health. The department of infectious disease control in the Institute of Public Health forwards AEFI data to WHO Uppsala Monitoring Centre [2] Although formal guidelines about adverse events 
following immunization have been developed since 2003 from the ministry of health, the real efforts to build a National AEFI surveillance system has started in 2008.

In Albania vaccination is mandatory by the low No.15/2016 "For the prevention and control of infectious diseases" which states that immunization is obligatory for children aged 0-18 years old. (Art.8) and it is one of the requisites for registration in school. [3]

The immunization schedule has changed over years and the actual schedule is operative since March 1, 2014. Children aged from 0-18 years old are immunized against 11 infectious diseases ; Tuberculosis (BCG), Hepatitis B (HepB), Diphteria, Tetanus, Pertussis (DTP-HepB-Hib, DTP, DT, Td), Haemophilus influenzae type b (Hib), Measles, Mumps, Rubella (MMR), Pneumococcal (PCV10) and Polio( IPV,OPV).

This study analyses and describes national passive surveillance data for adverse events following immunisation (AEFI) reported to the Institute of Public Health. Data are considered from 2008 till 2015 because during these years the immunization schedule has undergone various changes regarding new vaccines. On January 2009 it was introduced Hib as part of the combined vaccine DTP-HepB-Hib (Pentavalent) administered to children at 2, 4 and 6 months of age. On January 2011 it was introduced pneumococcal (PCV10) vaccine administered to children at 2, 4 and 6 months of age and on March 2014, inactivated polio vaccine (IPV) replaced the first two doses of oral polio vaccine (OPV), while the third dose of pneumococcal vaccine was shifted from 6 months to 10 months of age.

Monitoring of vaccine safety is critical component of vaccination programs; according to current guidelines in Europe, safety data are necessary to evaluate the benefit-risk profile of vaccines intended to be used on wide scale [4]. In these circumstances it is important to assess how the spontaneous reporting systems perform this function.

\section{Materials and Methods}

We performed a retrospective study on AEFI cases in Albania from 2008 -2015 and analysed the AEFI database in the Albanian Insitute of Public Health. The study has taken place from January 2016 to May 2016. In the individual case report we have the following information: Identification number of the subject, age, gender, birth place, date of vaccination, date of AEFI report, type of vaccine administered, place of vaccination, type of adverse event, treatment of the event and outcome of the event. Reports are recorded in the database regardless of causality and severity.

Data Analysis

Reported AEFI were extracted from the AEFI database as Microsoft Excel files. Dose-based reporting rate of AEFI per 100,000 vaccine doses was calculated as the number of reported AEFIs divided by the total number of administered vaccine doses.

Data for AEFI received by National adverse events surveillance system during the 8-year period 2008-2015 were analysed using IBM-SPSS ver.21 statistics program. The distribution of AEFI records was analysed by vaccine type, type of the event and treatment outcome. The Chi-square test $(\mathrm{x} 2)$ was used to compare the proportions and descriptive tests to evaluate frequencies. $95 \%$ confidence intervals were used for the mean values. Tests were considered statistically significant at $p<$ 0,05 .

\section{Limitations and strengths of the study}

Under-reporting is one of the most relevant limitations of every passive reporting system. Although the number of reports has increased in the last years it still remains low. The extent of under-reporting is not known exactly. It is difficult to compare background rates of adverse events after immunization with the background rates as the there is no control group (high vaccination coverage)

The quality of the data also is suspicious. Because of the reporter knowledge and professional capacity events reported may be inaccurate, non specific or missing. The reporter bias may affect reporting rates in passive reporting systems. [21] The reporter is influenced by new vaccines introduced in the market, time of the event occurrence and media awareness [22] Because of the multiple vaccine administration it is difficult to correlate the reported case with a single vaccine. The strengths of the study include the fact that we could calculate the reporting rates using doses of administered vaccines. 
Database was complete with the necessary information encoded by professional epidemiologist at the National Institute of public Health and the data received covered 8 years' time period; AEFI were ascribed to a vaccine without establishing a relationship of causality

\section{Results}

The total number of reports during these eight years was 99. The median number of AEFI reports per annum (2008-2011) was 12. The peak level of cases reported is observed in 2014. In 2015 it is observed a reduction of reported cases compared with that of 2014. Most of the AEFI reported were relatively mild and self-limited, with fever being the most commonly reported reaction. $44,5 \%$ of the cases received ambulatory treatment all of which recovered after treatment. $7 \%$ of the cases needed no treatment while $48,5 \%$ of the cases received hospital treatment from which $24.4 \%$ did not recover (12/99 cases) but were followed up at the moment of reporting.

\section{Vaccines}

Eight different vaccine types were reported in the 99 AEFI records received between 2008 and 2015 (Table 1). The vaccine with a high reporting frequency is is Pentavalent (DTP-HepB-Hib) $39 / 99(39,4 \%)$. In these cases pentavalent is the only suspected vaccine to be related to the event while there are 32 other cases in which pentavalent is co-administered with other vaccines. The immunization schedule imposes children to receive more than one vaccine at the same time. For this reason all administered vaccines at the same day are usually listed as 'suspected' of involvement in an adverse event[5]

Taking in consideration the WHO information sheets on rates of adverse events of each vaccine, it is very possible that most of the adverse events reported after pentavalent vaccine administration is related with DTP component. Similar to a WHO report on vaccine safety, OPV was not associated with any adverse events [6].

Table 1. Number of events per type of vaccine administered

\begin{tabular}{|l|l|l|}
\hline \multirow{2}{*}{ Vaccines } & Responses \\
\cline { 2 - 3 } & $\mathrm{N}$ & Percent \\
\hline DT & 3 & $3,00 \%$ \\
\hline HepB/DTP & 1 & $1,00 \%$ \\
\hline DTP & 12 & $12,10 \%$ \\
\hline PENTAVALENT & 39 & $39,40 \%$ \\
\hline PENTAVALENT/PCV & 22 & $22,20 \%$ \\
\hline PENTAVALENT/IPV & 6 & $6,10 \%$ \\
\hline PENTAVALENT/IPV/PCV & 4 & $4,00 \%$ \\
\hline PCV & 4 & $4,00 \%$ \\
\hline MMR & 4 & $4,00 \%$ \\
\hline Td & 4 & $4,00 \%$ \\
\hline Total & 99 & $100,00 \%$ \\
\hline
\end{tabular}

DTP containing vaccines

In Albania the pertussis component of the DTP vaccine is whole cell pertusis, therefore it is expected that adverse events related to whole cell pertussis develop after administration of DTP-containing vaccines. In general currently available combinations of DTwP with HepB and/or Hib do not result in adverse reactions that exceed, in frequency or severity, those seen with the same DTwP vaccine given alone [7]. For this reason DTwP vaccines are considered together. There were in total 12 cases reported in which DTP was the only vaccine suspected to be related to the event. All the adverse events reported as being related with DTP containing vaccines are consistent with DTP vaccine information sheet on observed rates of AEFI.[8] Mild adverse events following DTwP administration like rash, pain ant the injection site, hyperemia and inflammation were observed. Fever was the most common adverse event reported as being related to DTP-containing vaccines. In $7 \%$ of the cases DTP was the only vaccine administered, while in $21 \%$ of the cases Pentavalent was the only vaccine administered. Drowsiness was reported in two cases after Pentavalent vaccine. Anaphylaxis was reported in 5 cases where Pentavalent was the only vaccine administered while in 1 case where Pentavalent was co-administered with IPV vaccine. In one case, in a child aged 18 months toxic shock, convulsions, tremor, cyanosis and vomiting were reported after DTP vaccine administration. The infant was recovered after receiving hospital treatment. Encephalopathy was reported in two cases in which Pentavalent was one of the vaccines suspected to be related with the event. In one of these 
cases convulsions were reported and the child was followed up at the time of reporting but the information was insufficient to define it as Davret's Syndrome. More recent studies do not confirm an association between DTwP and acute encephalopathy. [8]

Table 2. Relation between number of doses and occurrence of AEFI after DTP-containing vaccines administration

\begin{tabular}{|l|l|l|l|l|l|}
\hline \multirow{2}{*}{ Vaccine } & \multicolumn{2}{|l|}{$\begin{array}{l}\text { Dose } \\
\text { number }\end{array}$} & \multirow{2}{*}{ Total } \\
\cline { 2 - 6 } & Dose 1 & Dose 2 & Dose 3 & Booster dose 1 & \\
\hline PENTAVALENT & 24 & 12 & 3 & 0 & 39 \\
\hline PENTAVALENT/PCV & 12 & 5 & 5 & 0 & 22 \\
\hline PENTAVALENT/IPV & 6 & 0 & 0 & 0 & 6 \\
\hline PENTAVALENT/IPV/PCV & 6 & 1 & 1 & 0 & $\mathbf{4}$ \\
\hline DTP & 2 & & & 0 & 12 \\
\hline HepB/DTP & 3 & 1 & 0 & 8 & 1 \\
\hline TOTAL & 0 & 0 & 1 & 0 & $\mathbf{8 4}$ \\
\hline
\end{tabular}

As indicated in the table most of the adverse events reported after DTP containing vaccines occur after the first dose administration (55,9\% of the cases reported to develop AEFI after DTP administration). A few events occur after first booster dose. The first dose of DTP containing vaccines is given at the age of 2 months. At this age immunization system is not fully developed in infants and there are still present antibodies from the mother. The frequency of local reactions tends to increase with the number of doses administered, while systemic reactions with the exception of fever may diminish with subsequent doses [9]

\section{Adverse Events}

Each AEFI case report lists one or more symptoms, signs, and/or diagnoses, so the number of the events exceeds the number of the cases. All the events were recorded using Brighton Collaboration case definitions terms. There were in total 158 reported adverse events after vaccine administration. The distribution and frequency of events listed in AEFI database for vaccines received between 2008 and 2015 are shown in Table 2. The most frequently reported AEFI was fever (54/99), followed by convulsions ( $14 \%$ of the cases).

According to WHO, Serious AEFI are considered those that result in death; are life-threatening; require in-patient hospitalization or prolongation of existing hospitalization; result in persistent or significant disability/incapacity, or; are a congenital anomaly/birth defect. [10] In our study no adverse events after immunization resulted in significant or persistent disability/incapacity nor were a congenital anomaly/birth defect. The cases considered as serious were those requiring hospital treatment or relevant medical conditions. Overall $30,3 \%$ (30/99) of AEFI cases received during 8 years of operation (2008-2015) meet the definition of serious. $20 \%$ of the cases defined as serious (6/30) didn't recovered after treatment compared to $10 \%$ in the non-serious events group ( $7 / 69)$. There were no deaths reported.

While non-serious were considered those cases which didn't need treatment (mild severity) or those which needed ambulatory treatment or event interfered with daily activities or loss of working hours(moderate severity)[10] Most of the reported events were mild and transient. They included pain at the injection site, urticaria like rash, local swelling, local redness, erythema and indurations at the injection site.

Table 3. Reaction categories frequency reported in the national AEFI database

\begin{tabular}{|l|l|l|}
\hline \multirow{2}{*}{ ADVERSE EVENTS } & \multicolumn{2}{|l|}{ Responses } \\
\cline { 2 - 3 } & $\mathrm{N}$ & Percent \\
\hline Anaphylaxis & 8 & $4,20 \%$ \\
\hline Toxic_shock & 1 & $0,50 \%$ \\
\hline Encephalopathy & 2 & $1,30 \%$ \\
\hline
\end{tabular}




\begin{tabular}{|l|l|l|} 
Abscess & 5 & $3,20 \%$ \\
\hline Fever & 54 & $34,20 \%$ \\
\hline Convulsions & 14 & $8,90 \%$ \\
\hline Genarlized urticaria & 7 & $4,40 \%$ \\
\hline Respiratory difficulties & 2 & $1,30 \%$ \\
\hline Cyanosis & 7 & $4,40 \%$ \\
\hline Vomiting & 4 & $2,50 \%$ \\
\hline Persistent Crying & 7 & $4,40 \%$ \\
\hline Hypothermia & 2 & $1,30 \%$ \\
\hline Syncope & 1 & $0,60 \%$ \\
\hline Hyperpyrexia & 1 & $0,60 \%$ \\
\hline Facial paralysis & 1 & $0,60 \%$ \\
\hline Limb pain & 3 & $1,90 \%$ \\
\hline Inflamation & 2 & $1,30 \%$ \\
\hline Hematoma & 1 & $0,60 \%$ \\
\hline Angioedema & 4 & $2,50 \%$ \\
\hline Other Reactions & 32 & $20,30 \%$ \\
\hline Total & 158 & $100,00 \%$ \\
\hline
\end{tabular}

Persistent crying. Some infants develop continuous crying which may be unaltered, inconsolable, and last for a number of hours. It is suggested that localized reactions may be a cause of persistent crying.[11] Persistent crying was reported after Pentavalent vaccine administration in 6 cases.

Convulsions were reported in 14 cases of which fever over $38^{\circ} \mathrm{C}$ was reported in 6 cases. While fevers are usually benign, an abrupt rise in temperature is a risk factor for febrile convulsions in susceptible children. [12]

Encephalopathy . There were in total 2 cases of encephalopathy reported after Pentavalent vaccination. One reported on 2010 ( 1 in 100,000 doses administered) and the other on 2012 ( 0.9 in 100.000 doses administered). Recent studies concluded that DTwP vaccination was not associated with an increased risk of encephalopathy [13]

Abscess at the injection site. There were 5 cases reported with abscess at the injection site after administration of Pentavalent vaccine in infants of 2 months of age and 1 case after Td administration in a 14 year old girl. In two of the cases fever over $38^{\circ} \mathrm{C}$ was reported and one of them was associated with persistent crying. Three of the cases were hospitalized and the outcome was positive except one that was followed up at the time of reporting.

Cyanosis was reported in 8 cases of which 6 were related with administration of pentavalent vaccine 1 with Td vaccine and 1 with DTP vaccine

Syncope was reported only in 1 case after pentavalent and IPV vaccine administration in a 2 month year old male infant. After perfusion and oxygen administration in the hospital the case was recovered.

Anaphylaxis. The average reporting rate of anaphylaxis per 100,000 administered doses was 1.07 cases. Although anaphylaxis is set apart from simple allergic reactions (e.g., urticaria, allergic rhinitis, asthma) by the contemporaneous implication of cardio-respiratory signs with mucosal and/or skin changes (urticaria, angioedema, etc.) there is still no consensus on exactly how to define anaphylaxis which has led to confusion and suboptimal diagnosis and treatment of this condition [14] The Council for International Organizations of Medical Sciences (CIOMS) differences between anaphylactic reaction, anaphylactic shock, and anaphylactoid reaction which is no longer used in current allergy terminology. CIOMS group specifies an "anaphylactic reaction" requiring the presence of just a single skin, respiratory, or cardiovascular symptom.[15]While Brighton Collaboration case definition avoids the term "anaphylactic reaction" due to its inference of a causal relation to a given exposure, referring to anaphylaxis only. [14] There are minor and major criteria which build level of diagnosis certainty. In the database there were only 8 cases defined as "anaphylaxis" although this might be misleading for the reasons specified above. In 6 of the cases Pentavalent vaccine was reported as being related with the event. In the other cases there are only one or a few of the signs and symptoms which although relevant does not constitute sufficient information for diagnosing the event as anaphylaxis. Generalized urticaria was observed in $7 \%$ of cases, rash was reported in $11 \%$ of the cases ,respiratory difficulties with tremor, cyanosis and vomiting was reported in 2 cases, cyanosis in 6 cases, 
angioedema in 4 cases and erithema in 3 cases. All the cases were recovered, except one which developed generalized urticaria and were reported as being followed at the moment

\section{Reporting trends}

The overall reporting rate was 2,1 cases per 100,000 doses administered. This is a low value compared with the reporting rates in Australia, 14,1/100,000 population in 2009 [16]; Zheijang Province,China, 9.2 per 100,000 doses in 2008 [17] ; USA, 5-7/100,000 doses [18] ; Oman, 10.8/100,000 doses [19] ; Emiglia Romagna, Italy 4,6/10.000 doses [20]

Mean value of reporting rates per year was 2,0625 $(95 \% \mathrm{Cl} ; 1,13 ; 3,03, \mathrm{p}=0,002)$

There is a significant increase in the reporting rates throughout years( $r=, 877, N=8, p=, 004)$. There is also significant difference between two groups ;serious and non serious $\left(x^{2}=15,3 p=, 000\right)$.It is observed an increase in the reporting of non serious adverse events through years, while the reporting of serious events remains constant. There has been two peaks of increased AEFI rates during these years, in the last quarter of 2009 due to the introduction of Pentavalent vaccine and in 2014 due to replacement of oral polio vaccine with inactivated polio vaccine. It is observed a trend toward reporting of mild events in contrast of more serious adverse events such as anaphylaxis and febrile seizures. The most frequent AEFI, fever over $38^{\circ} \mathrm{C}$ has no significant increase through years. There is neither an increase in cases of convulsions $(p=0.748)$.

The mean AEFI reporting rate for DTP-containing vaccines during the study period was 8,2 per 100,000 doses administered $(p=0,000, \mathrm{Cl}: 95 \%, 5,7 ; 10,7)$ compared with 21.7 per 100000 doses administered during the 2003-05 period in Oman.[19] The highest annual reporting rate was $12 / 100,000$ doses in 2014 , while the lowest one was $4 / 100,000$ doses in 2009 . There is a significant increase in the reporting rates for pentavalent vaccine throughout years $(p=0.035)$

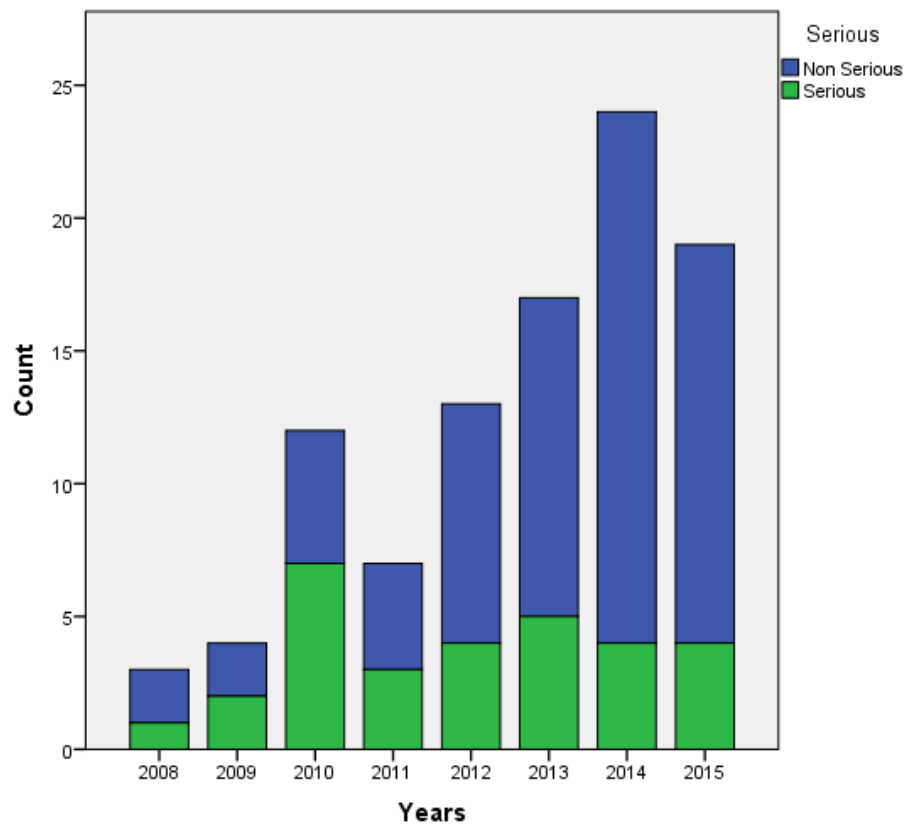

Figure 1. Reporting trends of AEFI throughout years

Most of the events occurred after administration of the first dose of the vaccine (51\% of the cases).A few occurred after administration of booster doses. 


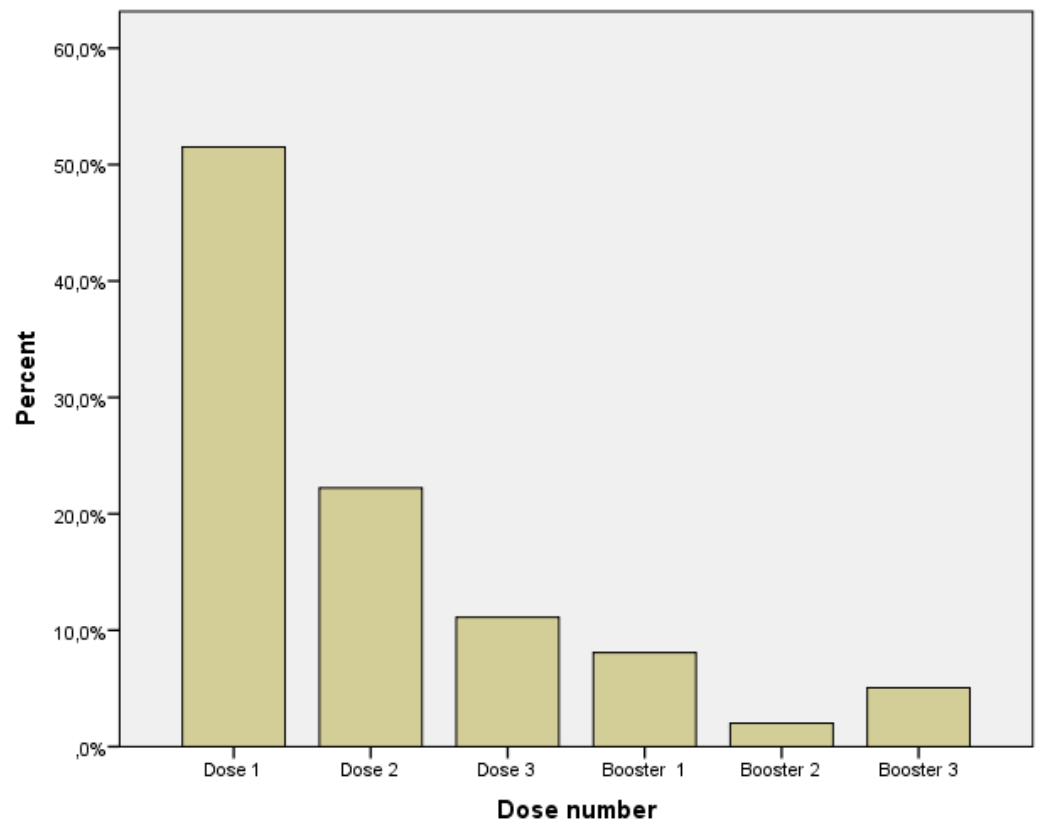

Figure 2.Relation between doses number and frequency of cases reported.

\section{Conclusions}

The majority of the adverse events reported during these 8 years are mild and non serious which somehow reflect the safety of vaccines in Albania. Overall the total number of reported events increased in the last 3 years (2013-2015) compared with the previous years. This increment can be explained by various factors. Introduction of new vaccines in the immunization schedule influences trends in reporting rates as the health care workers are more likely to report events related to a vaccine they are not familiar with. This is called Weber effect and this period is followed by a stabilization over time.[23] In our data this is reflected at the two peaks in 2009 after introduction of Pentavalent vaccine and 2014 after introduction of IPV vaccine. However the higher reporting rates reflect also the large number of activities undertaken by the $\mathrm{IPH}$ to train and encourage the reporting of adverse events following immunization.

Because of the limits of passive surveillance system mentioned above, information obtained by the AEFI database cannot be used to calculate the incidence rates of the adverse events, but it is useful to detect signals and generate hypothesis related to causality which should be further investigated by epidemiological studies. Underreporting still remains a problem, especially in rural areas making difficult interpretation of the data. In our data analysis it seems that DTP-containing vaccines are the vaccines showing the highest claims and still these are the vaccines with the highest occurrence of AEFI in Albanian children. This indicates the need for further studies and policy to pass from DTwP to DTaP

An AEFI reporting system is an integral part of an effective immunization program and analysis of data collated in the AEFI database are essential for immunization policy making. Paradoxically, when the immunization program is effective, the incidence of vaccine-preventable disease will drop, resulting in higher numbers of adverse events following immunization (AEFI) and its easier perception [24].Thus assessing vaccine safety issues effectively is essential to drop false concerns about vaccination and maintain at the necessary levels public trust on vaccination. Although the database is completed with the necessary information about data analysis, collection of more detailed and accurate information from AEFI individual reporting forms should be considered as quality of the data reported are critical for accurate analysis

The role of health-care workers is essential to a successful and well-conducted surveillance of AEFI.[25]Therefore the efforts of national immunization program should focus on qualification of the health care workers in this field to increase 
their active participation in surveillance of adverse events following immunization. The importance of professional qualification is reflected in the following results: $12 \%$ of the cases of AEFI did not recover after treatment suggesting the need for health worker training for management and diagnosing of an adverse event that follows immunization. It shows the need to improve and enrich the protocol of management of AEFI. 11 cases of rash and other cases with allergic reactions highlight the importance taking in consideration vaccines contraindications and assuring that the child is not allergic to any of the components of the vaccine. Two reports of injection site abscess reported with fever highlight the importance of sterile injection technique Differences between different countries regarding AEFI reporting are related to different reporting polices, data requirements, case definitions but they also reflect the data collection method, sensitivity of the surveillance system and compliance with the reporting.[26]

The objectives of the future include the implementation of an electronic method to report AEFI scaled up all-over the country and be accessed by all health centers in order to make the reporting of adverse events easier and faster. Legislative bases should be strengthened to mandate the reporting of AEFI. Developing tools to increase vaccine safety are is one of the most important priorities of IPH.

\section{References}

[1] Deepika M.et.al., 2007. Booster immunization with a hexavalent diphtheria, tetanus, acellular pertussis, hepatitis B, inactivated poliovirus vaccine and Haemophilus influenzae type $b$ conjugate combination vaccine in the second year of life: safety, immunogenicity and persistence of antibody responses. Vaccine 25:1055-1063

[2] World Health Organization 1999. Immunization safety surveillance: guidelines for managers of immunization programmes on reporting and investigating adverse events following immunization (WPRO/EPI/99.01). World Health Organization, Geneva, Switzerland: http://www.who.int/immunization_safety/publications/aefi/en/AEFI_WPRO.pdf

[3] Law No. 15/2016 "Over the prevention and treatment of infections and infectious diseases",Albanian Official Bulletin,No.46 pg.3069

[4] Brotherton JM, Gold MS. 2008. Monitoring vaccine safety: a critical component of every immunisation program. Med J Aust. 2008 Sep 1;189(5):243-4.

[5] Singleton JA, Lloyd JC, Mootrey GT, Salive ME, Chen RT. 1999. An overview of the Vaccine Adverse Event Reporting System (VAERS) as a surveillance system. Vaccine 17:2908 -2917.

[6] [World Health Organization. Dept. of Vaccines and Biologicals. 2000. Supplementary information on vaccine safety, Part 2: Background 9. rates of adverse events following immunization. Geneva, World Health Organization, 2000. WHO/V\&B/00.36 $105 \mathrm{p}$.

[7] Decker MM, Edwards KM, Bogaerts HH (2008). Combination Vaccines. In Plotkin SA, Orenstein WA, eds. Vaccines, 5th ed.Philadelphia, PA, WB Saunders Company, 2008:139-156.

[8] World Health Organization.2014.Information sheet on observed rates of vaccine reactions Diphtheria,Pertussis,Tetanus. http://www.who.int/vaccine_safety/initiative/tools/vaccinfosheets/en/

[9] Pollock TM, Miller E, Mortimer JY, Smith G (1984). Symptoms after primary immunisation with DTP and with DT vaccine. Lancet 2:146-9.

[10] Parrella A, Gold M, Marshall H, Braunack-Mayer A, Baghurst P. Parental perspectives of vaccine safety and experience of adverse events following immunisation. Vaccine 2013; 31:2067-74; PMID:23422146; http:/l dx.doi.org/10.1016/j.vaccine.2013.02.011

[11] J. Bonhoeffer et al.2004. Persistent crying in infants and children as an adverse event following immunization: case definition and guidelines for data collection, analysis, and presentation. Vaccine 22 (2004) 586-591

[12] Kohl KS. et al., 2004.Fever after Immunization: Current Concepts and Improved Future Scientific Understanding Clin Infect Dis. 2004 Aug 1;39(3):389-94. Epub 2004 Jul 9.

[13] Ray P, Hayward J, Michelson D et al. (2006). Vaccine Safety Datalink Group. Encephalopathy after whole-cell pertussis or measles vaccination: lack of evidence for a causal association in a retrospective case-control study. Pediatr Infect Dis J25:768-73

[14] Jens U. R"uggeberg a,b, et.al.,2007.Anaphylaxis: Case definition and guidelines for data collection, analysis, and presentation of immunization safety data_Vaccine 25 (2007) 5675-5684 The Brighton Collaboration Anaphylaxis Working Group

[15] Council for International Organizations of Medical Sciences (CIOMS) c/o World Health Organization. Definition and Application of Terms for Vaccine Pharmacovigilance: Report of CIOMS/WHO Working Group on Vaccine Pharmacovigilance.2012. 
[16] Mahajan D, Roomiani I, Gold MS, Lawrence GL, McIntyre PB, Menzies RI. 2010. Annual report: surveillance of adverse events following immunisation in Australia, 2009. Commun. Dis. Intell. 34:259-276

[17] Hu Y, Li Q, Lin L, Chen E, Chen Y, Qi X. 2013. Surveillance for Adverse Events following Immunization from 2008 to 2011 in Zhejiang Province, China Clin Vaccine Immunol. Feb;20(2):211-7.. Epub 2012 Dec 12. Institute of Immunization and Prevention, Zhejiang Center for Disease Control and Prevention, Hangzhou, China

[18] .Zhou W, et al., 2003. Surveillance for safety after immunization: Vaccine Adverse Event Reporting System (VAERS)-United States, 1991-2001. MMWR Surveill. Summ. 52:1-24 (Erratum, MMWR Morb. Mortal. Wkly. Rep. 52:113.)

[19] S. Al Awaidy, S. Bawikar,K.P. Prakash, B. Al Rawah and A.J. Mohammed. 2010. Surveillance of adverse events following immunization: 10 years' experience in Oman. EMHJ, Vol.16, No.5, 2010

[20] Maria Giulia Gatti et.al, Sorveglianza degli eventi avversi ai vaccini segnalati in Emilia-Romagna in età pediatrica (anni 2006-2011) e adulta (2010-2011).

[21] Callreus T.2010. Perceptions of vaccine safety in a global context. Acta Paediatr 2010;99:166-71

[22] Bentsi E, Hardy M, Koch J, Duclos P. 1995. Adverse events temporally associated with vaccines-1992 report. Can. Commun. Dis. Rep. 21:117-128

[23] Additional reports - childhood immunisation coverage. Commun Dis Intell 2008; 32: 288-9.

[24] Mônica P. L. Cunha, José G. Dórea, Rejane C. Marques, and Renata S. Leão . 2013.Vaccine Adverse Events Reported during the First Ten Years (1998-2008) after Introduction in the State of Rondonia, Brazil, Biomed Res Int. 2013; 2013: 853083.

[25] Parrella A, Gold M, Marshall H, Braunack-Mayer A, Baghurst P.2013. Parental perspectives of vaccine safety and experience of adverse events following immunisation. Vaccine 2013; 31:2067-74; PMID:23422146; http:// dx.doi.org/10.1016/j.vaccine.2013.02.011

[26] Brotherton JM, Gold MS.2008. Monitoring vaccine safety: a critical component of every immunisation program. Med J Aust 2008; 189:243-4; PMID:18759715 